\title{
Making Human-Computer Interaction Usable: Thinking from Healthcare Perspective
}

\author{
S.Sathya Franklin \\ Assistant Professor \\ Department of Computer Applications \\ Marwadi Education Foundation \\ Gujarat, India
}

\author{
R.Sridaran \\ Dean \\ Department of Computer Applications \\ Marwadi Education Foundation \\ Gujarat, India
}

\begin{abstract}
The effectiveness of Human Computer Interation depend upon certain important parameters such as usability and comfortability, which would encourage the end-users to get the services safely and more reliably. It becomes essential to understand the various usability factors under different perspectives which are highly essential to be considered by the healthcare system designers. As such, to the best of our knowledge, no such literatures of this kind are readily available. This paper categorizes the important usability factors and discusses some of the parameters that would make the interface effective and usable with certain quality parameters such as safety and reliability. Some of the problems of the interfaces from clinical perspective are also being discussed together with the strategies for improving the usability and in turn to meet the needs of the intended users.
\end{abstract}

\section{General Terms}

Human Computer Interaction, User-Interface Design, Usability Factors

\section{Keywords}

Clinical HCI, Interface Usability, HCI Testing

\section{INTRODUCTION}

Human-Computer Interaction (HCI) is the study of how people interact with the computers and to what extent computers are or are not developed for successful interaction with human beings [17]. When the users interact with a computer system, they do so via a User Interface. Interface is a part of the computer systems, through which the users interacts in order to use the system and achieve the goal [23].

This chapter begins with the discussion of the development process of $\mathrm{HCI}$ and then it focuses on how the HCI is being used in clinical field.

\subsection{HCI Development Process}

In this section, we briefly focus all about the design and development process of the 'soul' of the HCI, ie 'Interface'. The Fig. 1 depicts the process flow and the various phases which are involved in the HCI development process.

Based on the skill levels, the users of interface can be classified as Novice or First time users, Knowledgeable or Intermittent users and Expert or Frequent users. The five factors that will decide the user community can be stated as Time to Learn, Speed of performance, Rate of errors by users, Retention over time and Subjective satisfaction. Task identification plays a vital role in HCI to understand the domain of the interface and to observe and interview the users as well [17].

The user interface and the ways of interacting with computerbased systems are different across various systems [23]. Ben Shneiderman et al. [17] suggest that the appropriate interaction style will be chosen among various styles available such as Direct Manipulation, Menu Selection, Form-Fillin, Command Language and Natural Language and also provides the 8 golden rules of interface design that can be followed for effective design. Proper interface building tool can be used to build the prototype to mock-up the interface.

After the interface design, it should undergo Expert Review, which can be done by various experts in the domain. Useful guidelines and feedback can be received from the review process. The usability level of the interface can be measured by undergoing the Usability Testing (UT) with intended user community. If the UT is done during the development process, then the interface can be developed faster and the cost of redesign during later stages can be very much reduced. Then the hardware and software performance will be levied using the Acceptance Test (AT) and the user satisfaction level will be measured as well. If the interface is unable to meet the goals and acceptance criteria stated by the AT, then the interface will be redesigned. The process will be repeated until an acceptable interface is being developed [17].

\begin{tabular}{|c|c|c|c|c|c|c|}
\hline \multicolumn{7}{|c|}{ QUALITY ASSURANCE PHASE } \\
\hline $\begin{array}{l}\text { Error } \\
\text { Prevention }\end{array}$ & $\begin{array}{l}\text { Expert } \\
\text { Review }\end{array}$ & $\begin{array}{l}\text { Usability } \\
\text { Testing }\end{array}$ & $\begin{array}{l}\text { Acceptance } \\
\text { Testing }\end{array}$ & $\begin{array}{l}\text { User } \\
\text { Survey }\end{array}$ & \multicolumn{2}{|c|}{$\begin{array}{l}\text { Risk } \\
\text { Assessment }\end{array}$} \\
\hline \multicolumn{7}{|c|}{ DESIGN PHASE } \\
\hline $\begin{array}{l}\text { Interaction } \\
\text { Style }\end{array}$ & \multicolumn{2}{|c|}{8 Golden Rules } & \multicolumn{2}{|c|}{$\begin{array}{l}\text { Interface } \\
\text { Building Tools }\end{array}$} & \multicolumn{2}{|c|}{ Prototyping } \\
\hline \multicolumn{7}{|c|}{ CONCEPT PHASE } \\
\hline $\begin{array}{l}\text { Interface } \\
\text { Goal }\end{array}$ & $\begin{array}{l}\text { User Group } \\
\text { Identification }\end{array}$ & $\begin{array}{l}\mathrm{HCl} \\
\text { Theories }\end{array}$ & $\begin{array}{l}\text { Design } \\
\text { Guidelines }\end{array}$ & $\begin{array}{l}\text { Specific } \\
\text { Tools }\end{array}$ & ation & $\begin{array}{l}\text { Legal } \\
\text { Issues }\end{array}$ \\
\hline
\end{tabular}

Fig. 1 HCI Development Process Flow

\subsection{Role of HCI in Healthcare}

In recent days, it is obvious that the healthcare industry has more rapid influence and development with HCI. It is very well known that the current healthcare environment utilizes to the higher extend of the computer software in medical devices, which are being used very commonly in departments such as operating rooms, emergency rooms, patient units, Xray departments, laboratories, critical care facilities, clinics and home. These devices are a diverse group of products that ranges from simple items such as weighing machine to complex devices such as heart by-pass machines [4] [6].

Computers are introduced in healthcare industry mainly to improve the precision in the diagnostic process and to make the easy and efficient process to be readily available 
worldwide [11]. High quality and well designed medical devices are necessary to provide safe and effective clinical care for patients as well as to ensure the health and safety of device users [4]. Though many devices are successful, some of them will be with poor performance and may produce erroneous result. Errors may challenge the strength and quality of the interface.

To improve the quality of the medical devices, it is not enough of strengthening the medical computing capabilities, but it is necessary for the practitioner to have a wide knowledge and awareness about the device [11]. Ensuring a good quality and usability will only bring great success in the digital world [12] [22].

In this research, the usability factors of HCI in healthcare are viewed from the perspective of user- one who interacts with the interface and the interface- through which the user interacts with the computer. This paper reveals the problems of medical interface and the possible solutions to improve the usability and hence to make it useful.

\section{RELATED WORK}

Nowadays, the need of modernizing the healthcare industry and the accuracy in diagnosis process are very much essential. Hence, the researchers and academicians were motivated to do research on implementing various strategies of $\mathrm{HCI}$ in healthcare.

Patient Safety: The patient safety was dealt in many research works. Jiajie Zhang et al. [5] discussed about improving the patient safety in healthcare. David Wood [9] revealed some of the human factors which will lead to the system failure and patient safety from the HCI technician's perspective. The research by Jim Nindel et al. [6] found the reason for interface failure and some of the useful ways to implement the HCI testing process in healthcare. Dick Sawyer [10] suggested the guidelines to manufacturers to improve the safety of the medical device. Although the patient safety is important, the strategies to improve the overall usability of the design are not found in any of these research works.

Data Maintenance: Andreas Holzinger et al.[13] has considered the medical data maintenance in their research work and revealed the strategies to make the data useful in some particular medical applications. The Dan Armijo et al. [26] discussed the effective data management through electronic health record (EHR) from the storage perspective. But the useful and efficient data management from the interface aspect was not discussed in any of the research.

In spite of much discussion about the influence of HCI in healthcare, there are very few researches talking about the usability improvement from the perspective of interface design. Also, some of the surveys discusses the problems of HCI design in healthcare and hardly reveal the suggestions to make it useful. Many researchers discussed about the patient safety, efficient data management and effective hardware and display in their paper, but the strategies to improve the overall usability with the factors of interface and operator's perspective, are not found in any of the surveys.

A research is required considering the problems of $\mathrm{HCI}$ in healthcare and to give the possible solutions to improve the usability. This proposed research approaches the healthcare field by considering the following components such as software, hardware and the HCI operators and lists the problems and the possible recommendations to make the interface useful in healthcare.

\section{USABILITY IMPROVEMENT OF HCI IN HEALTHCARE}

The proposed research suggests the strategies to improve the usability of the interface from the HCI operator as well as interface design perspective. Fig.2 shows the schematic diagram highlighting the factors that are to be considered in order to enhance the effectiveness of the interface in clinical field. While viewing the HCI from the perspective of healthcare, the two major key elements that can be identified and stated as:

- 'Interface'- medium through which the human interacts with the computer

- 'Operator'- the user who interacts with the interface

These elements are elaborately discussed further with various factors that may affect the usability of the interface directly or indirectly.

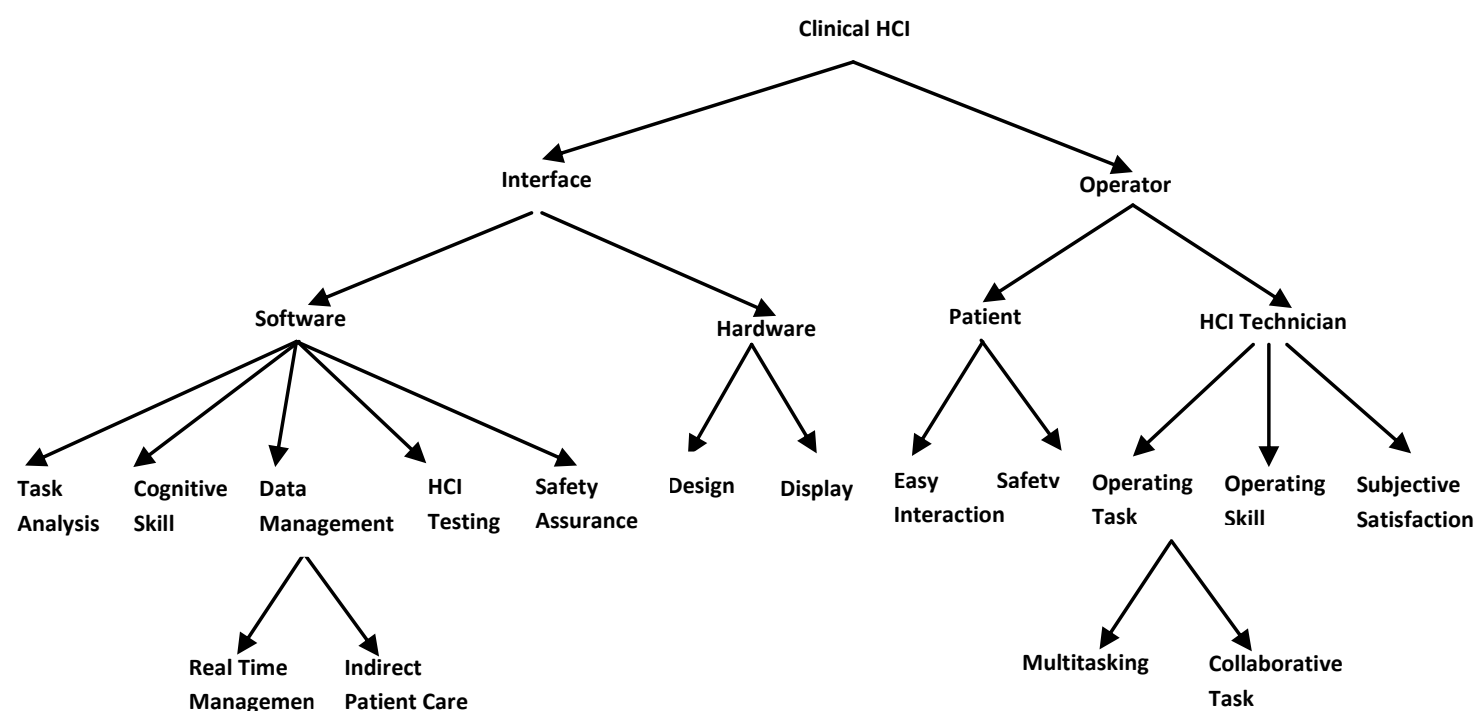

Fig. 2 Usability Factors of HCI from Healthcare Perspective 


\subsection{Interface}

The media through which the user (Human) interacts with the medical device (Computer) is known as 'interface'. The two major components that constitute an interface of the medical devices can be stated as the 'Software' and the 'Hardware'. The following session discusses some of the factors related to software and hardware that affects the usability of the medical device.

\subsubsection{Software}

Software component of the interface plays a vital role to improve the usability of the medical interface. It is mainly used in medical devices to monitor and control the patient's diagnosis and treatment process. Some of the key factors such as task analysis, cognitive skills, data management, testing and safety assurance, that can be considered to enhance the overall usability of an interface, are dealt in the subsequent sections.

\subsubsection{Task Analysis}

Collecting and understanding the user's task or interface goal is very important and tedious job in the concept phase of the interface as shown in Fig.1. For an efficient interface, there is no much deviation between the expected and the actual results. A computer system that has developed without a good knowledge of the users, will accomplish the task partially but will not fulfil completely. The major task of an interface is not merely representing the procedures required, but also making the end result to match with the task [23]. Similarly, the other main goal of a design task is to assist the users in orientation and navigation. Axel Platz et al. [8] suggest that every interface should satisfy the following tasks completely:

- Providing the awareness to the users to make them understand their actions and to proceed towards their task (Orientation).

- Conveying the sequence of actions to be carried out by the user (Navigation).

The interface should ensure that the novice users are not lost in half the way while operating the interface [8]. The design should meet the needs, capabilities and limitations of users, rather than expecting the users to adapt the design to make it useful [5].

\subsubsection{Cognitive Skill}

The cognitive skill of the interface can be viewed as the ability to think from the user's perspective. HCI designers should be able to visualize and 'think' about the interface and should have the knowledge on user's familiarity on the interface [19]. Moiseeva et al. [11] feels that the computer neither replace a physician nor 'thinks' instead of him, but it 'thinks' with him. The interface is expected to think and behave like a human to make it more useful. The 'Anthropomorphic design' [17] ensures that the interface can behave like a human and will be able to give relevant feedback, which could be accepted or denied later by the users [11]. Since each interface is different, depending on the design and performance of the interface, it can be predicted that the system will either be usable or unusable for users [23]. Hence, a strong cognitive skill is required from the designer's end to make the interface useful.

According to Andre Kushniruk et al. [24], some of the ways to impart the cognitive skills for improving the healthcare information systems are by:
- Providing knowledge about what typical users of the systems can and cannot be expected to do.

- Identifying and explaining the nature and cause of user problems.

- Characterizing the problem solving and decision making processes of healthcare workers.

- $\quad$ Suggesting useful information for system re-design and improvement.

- $\quad$ Providing models and frameworks for conducting HCI research in Healthcare.

\subsubsection{Data Management}

Research in HCI and information management should work together to develop methods that help people to identify, extract and understand useful information from data [30]. Computerization of information management is an effective way to help medical staff to perform their job better in clinical environment [29].

In a computer-supported medical environment, the relevant data regarding the patient should be collected well in advance, so that it will be useful for an efficient diagnosis process [8]. This will help the physician to make a suitable prescription during treatment process and particularly in emergency department where computer-based support is very much unavoidable. Hence, the storage and retrieval process of medical data should be done carefully. During the data entry process of the medical record, the interface should ensure that all the mandatory data are being filled and that no required fields are left blank [16][29].

The Electronic Health Record (EHR) is one of the best practices used to construct and maintain patient's record and an effective way to query large amount of available medical data. EHRs are Clinical support tools with the potential to reduce the burden on clinical memory and cognition while improving efficiency in workflow and effectiveness in healthcare quality. The interface should also ensure that the privacy and integration of the medical records will not be getting affected [25][26][29].

The record maintenance strategies can be divided into two categories such as real time management of the emergency service and indirect patient care and resource management. The real time management of the emergency service includes the patient registration, entry of diagnosis data, test requests, and nursing tasks. The indirect patient care and resource management focuses on definition of clinical protocols, extraction of statistics and management of access rights [29]. The challenge is to bring HCI and information retrieval to work together and to achieve the maximum benefits, so that it will improve the healthcare field even more [30].

\subsubsection{HCI Testing}

HCI testing is the process of analysing and measuring the performance and quality of the interface. The quality of the interface will be diminished by poor performance and errors. HCI testing will improve the interface quality and reduce serious errors, and in turn will increase the user satisfaction and usability. Using the HCI testing, the interface failure can be very much reduced. For avoiding the interface failure, the designers can test the design at any intermediate level without having the need to wait until finish it completely [20]. Jim Nindel et al. [6] discuss that, in order to avoid the interface failure, the HCI testing is very much essential at the early 
stage of the interface development life cycle. The challenge of the HCI developers is to determine the criteria for 'sufficient' HCI testing.

The patients and medical professionals can be the common potential users of medical devices. Hence, it is ideal to consider both types of users in the HCI testing process [6]. The Food and Drug Administration (FDA) published the guidelines for interface design and usability testing and has produced an education article that specifically covers usability issues [5]. The HCI designers can consider those guidelines and regulations in the development process for improving the usability of the medical device.

\subsubsection{Safety Assurance}

Safety is considered as a major quality factor which is required by majority of the users and very much essential for a medical interface. The medical device should ensure that the safety is preserved while the users operate the interface. The Journal of the American Medical Association [3] has reported that the computers increase the error risk in clinical medical devices and it added that the computerized medical devices are not designed to reflect the real life scenario which is being followed. FDA data shows that nearly half of the withdrawals of medical device were due to the poor user interface design [5]. It is the goal of the interface designers to verify 'what if' anything unexpected happens and how the system responds in that situation [6].

For legal and privacy concerns many unforeseen incidents are under-reported and are often not discussed widely by hospital authorities. The medical device operators hardly reveal the error and negative outcomes to the patients [1]. The evolving technologies need to focus and facilitate the safety and error free delivery of healthcare [13][14].

Richard A Schrenkar [14] concluded that the only way to prevent the medical interface accidents is either to build a complete HCI tested software or not to use the computeraided device at all [14]. Producing error free software is highly a challenging task. To ensure the safe and reliable user interfaces, not only an error-free interface but an errortolerance interface is also very much required [28]. Some of the key points to be considered to come out with a safer design are:

- Organize the interface components visible

- Make the orientation and navigation processes as far as simple

- Must not force the user to load many things to their memory

- Not to expect more vigilance from the user

- $\quad$ Reversing an error should be easy

Hence, it can be stated that the software component of the medical interface can be improved by proper understanding of the task, incorporating the cognitive skill to the interface, the efficient data management, proper testing and performance measurement and assurance of the safety to the operator. At this moment, it is necessary to discuss the usability factors to improve the hardware component of the interface also.

\subsubsection{Hardware}

Hardware deals with the overall design and peripheral devices of the computer that is being used for the interaction. It is just the device design but without which human may find it much harder to interact or understand the design of the interface. The design of the medical equipment should be provided in such a way that it can be marketed faster and easier to interact. The countries like India, China, and Brazil are investing huge amount in healthcare industry for demanding affordable, robust, and reliable medical devices [15].

Design of the devices and display characteristics are the two aspects of hardware design that can be considered to improve the usability. They are discussed further in the following session

\subsubsection{Design}

The design of the medical device matters much when considering the improvement of the usability. Some of the toolkits are available that assist researchers and designers to create new effective hardware [21]. Medical interface designers are exploring new technologies to improve the diagnostic and monitoring capabilities of next-generation devices. The new design technology should offer and focus on complex functionality in a compact size, high accuracy and reliable operation. The other factors are being flexibility, integration, cost savings, and offering power reduction also [15].

Interaction devices such as keyboard and pointing devices play a major role in hardware side of the interface. The international standards for hardware interface design can be adapted to design effective hardware and interaction devices [27]. The keyboard needs to have a good mechanical design and efficient placement of letters for effective use. The key labels should be large, meaningful and permanent to improve the usability of the device. The function keys can be programmed for frequent actions, which in turn reduce the number of keystrokes and errors. Error rates can be reduced by selecting appropriate pointing devices with respect to the interaction tasks [17].

Hardware design should ensure the reduction of learning time, cost, and space requirement so that the user satisfaction will be increased. The design should be adaptable by the left and right hand users also. The positive and useful information in the form of sound can be given in some situations so as to confirm some serious actions, to offer warning messages, and to aid visually impaired users [17].

Along with the discussion of qualitative parameters, it is good considering the quantitative parameters also for the improvement of hardware design. Fitt's law [2] is very well suitable to explain the quantitative requirement to improve the usability of a hardware design of an interface. He observed that the action of pointing to a target object could be measured and predicted mathematically. It is stated as shown below:

$$
\mathrm{T}=\mathrm{a}+\mathrm{b} \log _{2}(2 \mathrm{D} / \mathrm{W})
$$

Where, $\mathrm{T}$ is the time taken to interact with an interface widget, $\mathrm{a}$ and $\mathrm{b}$ are coefficients, $\mathrm{D}$ is the distance between the interface and the operator, and $\mathrm{W}$ is the width of the target widget.

With the above equation, the Fitt's law concludes that the size of the target object should be larger and the distance between the interface and the HCI technician should be minimal in order to get the better result, and in turn to reduce the time taken to accomplish the task. 


\subsubsection{Display}

The display parameter determines how effectively the interface can be viewed by the operators. The quality of the display should be appropriate enough to attract and satisfy the potential users. It is mainly determined by the display resolution and correctness of colours in the interface. Even though the usage of more colours appears to be attractive, at times it may not give emphasis to the correct widget properly and may look clumsy. Hence, choosing the appropriate colour will also affect the user satisfaction to some extent. The blur colour reduces the importance of the interface and may not convey its purpose. Hence, limiting the number and amount of colour will be advisable and should go with universal colour code standard. The refresh rate is another factor that should be sufficient enough to allow animation and video with a higher quality [17]. The expected problems and concerned recommendations related to display are listed in the Table 1 in Appendix.

After considering the usability factors from the interface side, it is necessary to discuss few more factors that are required to improve from the operator's side too. Hence, the following section discusses the various ways of improving the usability of the medical device from the user's perspective. The usability factors also have been discussed in detail by us from operator's perspective [33], whose summary appears in the subsequent sections.

\subsection{Operator}

The operators are considered to be the human users who directly interact with the interface. The two major potential users of medical interfaces can be stated as Patient and HCI technicians.

\subsubsection{Patient}

The user convenience and their safety are the two major factors that are sufficient to convince the patient, and in turn to achieve satisfaction where User Convenience can be stated as the combination of easy interaction and faster learning. Users should constantly get the informative feedback from the interface for their every piece of action to make the interface effective. The interface with a smaller screen will be a problem for some users and they may not get the exact information on the fact that what the interface is conveying.

The Patient's Safety is another healthcare discipline that emphasizes on prevention of medical errors that may often lead to some serious negative situations. The design which fails to fit the requirement and standards of HCI will put the patient's safety at risk. Safety and effectiveness of the medical device can be achieved only if the HCI designers consider the coordination between the operating environments, user capabilities, stress levels and device design during the development stage. The other significant operator in this scenario is the HCI technician whose usability factors are listed below.

\subsubsection{HCI Technician}

Knowledge on the operating task, the skill to operate the interface and their subjective satisfaction are the major factors to be considered from the HCI technician's perspective to improve the usability.

The technicians are expected to have adequate knowledge about Operating Task of the interface. A well designed interface will direct the user in a proper way to accomplish the task whereas a poorly designed one will challenge the technicians with unexpected behaviour.

An Operating Skill is required by the technician to a great extent to understand and operate the features and functions of the interface effectively. They should understand the requirement and manufacturer's intentions for efficient usage of devices. An improper user interface can lead to error and operating inefficiencies even it is operated by a well-trained and competent user.

The Subjective Satisfaction of the technician should be considered seriously to improve the usability because every operator prefers that the design of the devices should be comfortable and have to go with the regular work flow. It is not enough that the users know to use the interface, but they must be comfortable to operate the device. The design should consider the basic capabilities of users such as strength, memory, reach, vision and hearing of the users to attain satisfaction. It should ensure that the brightness of visual effects is sufficient in all situations and make sure that the abbreviations, symbols and text are consistent with the instruction manual. [33].

\section{CONCLUSION \& FUTURE WORK}

Usability factors require the consideration of human's convenience, Cognitive skill and feedback for the successful implementation of UI for healthcare systems. An efficient Usability Testing is very much essential for better use of the interface. Healthcare systems with improved HCI would benefit the industry in terms of quality of service and throughput. This paper has attempted to categorize various such factors under qualitative basis. However our future work would involve certain significant quantitative factors related to these qualitative factors to address the needs of next generation healthcare systems to improve the usability of the interface.

\section{REFERENCES}

[1] ChitraAcharya, Harold Thimbleby and Patrick Oladimeji, "Human Computer Interaction and Medical Devices", proceedings of the $24^{\text {th }}$ BCS Conference on Human Computer Interaction, Oct 2011.

[2] The Six Revisions Website [Online] Available: http://sixrevisions.com/usabilityaccessibility/improvingusability-with-fitts-law/

[3] The Journal of the American Medical Association Website [Online] Available: http://jama.jamanetwork.com/journal.aspx.

[4] Jennifer L Martin, Beverley J Norris, Elizabeth Murphy, John A Crowe, "Medical Device Development: The Challenge for Ergonomics", Applied Ergonomics, vol.39, pp.271-283, 2008.

[5] Jiajie Zhang, Vimla L Patel, Todd R Johnson, Philip Chung, James P Turley, "Evaluating and Predicting Patient Safety for Medical Devices with Integral Information Technology", Advances in Patient Safety, vol.2, pp.323-336.

[6] Jim Nindel-Edwards and Gerhard Steinke, "Integrating Human Computer Interaction Testing into the Medical Device Approval Process", Communications of the IIMA, vol.9, Issue 2, 2009 
[7] Luca Pazzi and Marco Pradelli, "A State-Based Systemic View of Behaviour for Safe Medical Computer Applications", $21^{\text {st }}$ IEEE International Symposium on Computer-based Medical Systems, 2008.

[8] Axel Platz and Claus Knapheide, "Interactive Design using the Example of a Complex Medical Application", Inernational Journal of Human Computer Interaction, vol.12, pp.431-440, 2000.

[9] David Woods, "Behind Human Error: Human Factors Research to improve Patient Safety", Human Factors and Ergonomics Society.

[10] Dick Sawyer, "Do it by Design: An Introduction to Human Factors in Medical devices", publication by office of the Health and Industry Programs.

[11] N I Moiseeva and V VUsov, "Some Medical and Mathematical Aspects of Computer Diagnosis", Proceedings of the IEEE, vol.57, No.11, pp.1919-1925, Nov 1969.

[12] Andreas Holzinger, Harold Thimbleby and Russell Beale, "Workshop HCI for Medicine and Healthcare (HCI4MED), $22^{\text {nd }}$ British HCI group Annual Conference, Sep 2008.

[13] Andreas Holzinger, Harold Thimbleby and Russell Beale, "Human-Computer Interaction for Medicine and Healthcare (HCI4MED): Towards Making Information Usable", International Journal of Human-Computer Studies, vol.68, pp.325-327, 2010.

[14] Richard A Schrenkar, "Software Engineering for Future Healthcare and Clinical Systems", IEEE Computer Society, vol.39, pp.26-32, 2006.

[15] The Medical Electronics Design Website [Online] Available: http://www.medicalelectronicsdesign.com/designmedresources-human-computer-interfaces-hci

[16] Mikael Palmblad and Brian Tiplady, "Electronic Diaries and Questionaires: Designing User Interfaces that are Easy for all Patients to Use", Quality of Life Research, vol.13, pp.1199-1207, 2004.

[17] Ben Shneiderman and Catherine Plaisant, "Designing the User Interface: Strategies for Effective Human-Computer Interaction", $4^{\text {th }}$ Ed., Pearson Addison-Wesley, 2004.

[18] Dag Svanas, Ole Andreas Alsos, and Yngve Dahl, "Usability Testing of Mobile ICT for Clinical Settings: Methodological and Practical Challenges", International Journal of Medical Informatics, vol.79, pp.e24-e34, 2010 .

[19] Eric J Horvitz, "Innovations at the Human-Computer Interaction: A Medical-Informative Perspective", Proceedings of Medicine meets Virtual Reality: Applications for 3-D Multimedia Technology in the Health Sciences, June 1992.

[20] James A Landay and Brad A Mayers, "Sketching the Interfaces: Towards More Human Computer Interaction Design”, IEEE, pp.56-64, March 2001.

[21] The Media Computing Group Website [Online] Available: http://hci.rwth-aachen.de/toolkits
[22] David A Vogel, "Software User Interface Requirements for Medical Devices", Medical Device and Diagnostic Industry, Aug 2007.

[23] The Elsevier Direct Website [Online] Available: http://www.elsevierdirect.com/companions/97801208843 60/casestudies/ Chapter_01.pdf

[24] Andre Kushniruk and Joseph Kannry, "Human Aspects of Healthcare Information System", Medical Sciences, vol.2

[25] Mark Ginsburg, "Pediatric Electronic Health Record Interface Design: The PedOne System", Proceedings of the $40^{\text {th }}$ Hawaii International Conference on System Sciences", 2007.

[26] Dan Armijo, Cheryl McDonnel, and Kristen Werner, "Electronic Health Record Usability: Evaluation and Usecase Framework", AHRQ Publication No.09, Oct 2009.

[27] The International Journal of Human Computer Studies Website [Online] Available: http://www.sciencedirect.com/science/article/pii/S10715 81901904835.

[28] Armin Janb, Wolfgang Lauer, Chuembou Pekam, Klaus Radermacher, "Using New Model-Based techniques for the User Interface Design of Medical Devices and Systems", IGI Global, Chapter 10, pp.234-235, 2011

[29] Teh Amouh et al., “ Versatile Clinical Information System Design for Emergency Departments", IEEE transactions on Information Technology in Biomedicine, vol.9, No.2, pp.174-183, June 2005.

[30] Andreas Holzinger, "Interacting with Information Challenges in Human-Computer Interaction and Information Retrieval".

[31] C C M Hummels and C J Overbeeke, "Designing and Testing Human Computer Interaction: A Case Study in Virtual Clay Modelling", Conference Proceedings of the $31^{\text {st }}$ International Symposium on Automotive Technology and Automation (ISATA), pp.27-34, 1998.

[32] Ping Yu and Hui Yu, "Lessons Learned from the Practice of Mobile Health Application Development", IEEE Computer Society, Proceeding of the $28^{\text {th }}$ Annual International Computer Software and Applications Conference, pp.58-59, 2004.

[33] R Sridaran, S Sathya Franklin, "Analysis of Usability Factors of HCI Operators for Healthcare Applications", National Conference on Emerging \& Innovative Trends in Computer Science (NCEITCS-2012), pp.1-4, 2012, ISBN: 978-81-924922-0-9.

\section{AUTHOR PROFILE}

S.Sathya Franklin is an Assistant Professor in the Department of Computer Applications at Marwadi Education Foundation's Group of Institutions, Rajkot, Gujarat. He has obtained his Masters Degrees in Computer Applications and Electronics. He is currently pursuing his research in the area of Human Computer Interaction (HCI) for healthcare applications.

R.Sridaran is currently the Dean of the Department of Computer Applications at Marwadi Education Foundation's Group of Institutions, Rajkot, Gujarat. He holds the Masters 
Degrees in Computer Applications and Business Administration and a $\mathrm{PhD}$ in Computer Science. His research interest includes Design Patterns, Cloud Computing and Human Computer Interaction (HCI).

\section{APPENDIX}

Table 1. Problems with the display characteristics and recommendations

\begin{tabular}{|c|l|l|}
\hline Feature & \multicolumn{1}{|c|}{ Expected Problems } & \multicolumn{1}{c|}{ Recommendation } \\
\hline Font Size & Small font may be interpreted as smaller target & Usage of 12 pt for Moderate attention \\
\hline Language & Chaos when the interface comes to universal & Common Language is preferable \\
\hline Controls & User may ignore if the control is in a wrong place & Maintain the Consistency and Standard \\
\hline Colour & $\begin{array}{l}\text { Blur colour reduces the important and usage of } \\
\text { many colours will be clumsy }\end{array}$ & $\begin{array}{l}\text { Limit the number and amount of colour and can } \\
\text { go with Colour Code standard }\end{array}$ \\
\hline Symbols & May have different meaning in different culture & Design only with Standard Symbols \\
\hline $\begin{array}{c}\text { Numeric } \\
\text { Entry }\end{array}$ & May not be possible with high precision values & Usage of Slider Control \\
\hline $\begin{array}{l}\text { Feedback } \\
\text { Messages }\end{array}$ & May be blaming and not comprehensive & Can be Pleasing and Explanatory \\
\hline Navigation & $\begin{array}{l}\text { May be Clumsy, Confusing and Chance of } \\
\text { getting lost }\end{array}$ & $\begin{array}{l}\text { Maintain a Consistent format and must direct } \\
\text { towards the proper completion }\end{array}$ \\
\hline Table Data & $\begin{array}{l}\text { Larger table which exceeds more than one page } \\
\text { may have no heading details }\end{array}$ & $\begin{array}{l}\text { Ensure that Column Headings are visible in all the } \\
\text { displayed sections of the Table }\end{array}$ \\
\hline
\end{tabular}

